\title{
Atopieprävention: Stillen plus Probiotika
}

\author{
Zur Primärprävention von Allergien wird Müttern atopiegefähr- \\ deter Kinder das Stillen ihrer Säuglinge empfohlen. Eine finnische \\ Studie untersuchte, ob die regelmäßige Zufuhr von Probiotika bei \\ den Müttern das Atopierisiko der Kinder weiter minimieren kann.
}

$\mathrm{D}$ urch die Muttermilch wird der unreife Säugling immunologisch geschützt. Eine entscheidende Rolle spielt dabei der Transforming Growth Factor $\beta$ (TGF- $\beta$ ): Er fördert die IgAProduktion und die Ausbildung der oralen Toleranz. Eine Steigerung der TGF- $\beta$-Produktion könnte auch einer der Mechanismen sein, durch die Probiotika allergische Entzündungsreaktionen mildern bzw. einer Allergie vorbeugen. Eine Weitergabe von TGF- $\beta$ durch die Muttermilch ist gesichert.

In der doppelblinden Untersuchung erhielten schwangere Frauen aus Atopiefamilien vier Wochen vor der geplanten Entbindung und während der gesamten Stillperiode entweder den probiotischen Bakterienstamm Lactobacillus rhamnosus GG (LGG) oder
Plazebo. Die Auswertung der Daten von 62 Mutter-Kind-Paaren ergab eine protektive Wirkung der Probiotika: Der TGF- $\beta$-Wert lag unter Verum signifikant höher als unter Plazebo $(\mathrm{p}=$ 0,018). Umgekehrt war das Risiko, in den ersten zwei Lebensjahren ein atopisches Ekzem zu entwickeln, bei den Probiotika-Kindern signifikant vermindert ( $15 \%$ vs. $47 \%$, p = 0,0098). Kriterien für das Vorliegen eines atopischen Ekzems waren Juckreiz, typisches Erscheinungsbild und Lokalisation der Hautläsionen sowie der schubförmige Verlauf mit drei oder mehr Krankheitsepisoden.

\section{Fazit}

Die kontinuierliche Gabe von probiotischem LGG vor der Geburt und wäh-

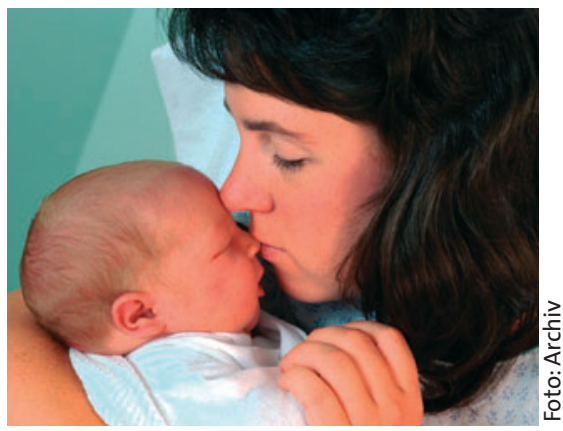

Muttermilch für das Kind und Laktobazillen für die Mutter- sieht so eine sinnvolle Prävention des atopischen Ekzems aus?

rend der Stillperiode scheint die immunmodulatorische Wirkung des Stillens zu unterstützen. Atopiegefährdete Kinder sind in den ersten beiden Lebensjahren auf diese Weise besser vor der Entwicklung eines atopischen Ekzems geschützt als bei alleinigem Stillen.

Rautava S et al. Probiotics during pregnancy and breast-feeding might confer immunomodulatory protection against atopic disease in the infant. J Allergy Clin Immunol 2002; 109: 119-21

\section{SIT kann Allergiekarriere bremsen}

\section{Wie entwickeln sich saisonale allergische Rhinokonjunktivitis und Asthma von Kindern, wenn die kleinen Patienten frühzeitig mit spezi- fischer Immuntherapie (SIT) behandelt werden? Um mögliche Lang- zeiteffekte zu finden, erhob man von den Teilnehmern einer klini- schen Studie sechs Jahre nach Therapieende erneut prospektiv Daten.}

$\mathrm{V}$ erglichen wurde eine vor 6 Jahren aktiv therapierte Gruppe von 14 Patienten (präsaisonale Immuntherapie mit Allergovit ${ }^{\circledR}$ während drei aufeinander folgender Jahre) mit einer Gruppe von 10 Patienten, die damals nur eine Standardtherapie (medikamentöse Behandlung) erhalten hatten. Die Patienten wurden gebeten, Symptome und Medikamentenbedarf während der Pollensaison zu dokumentieren.

Signifikante Differenzen beim Symptom-Score lieferten einen Beleg für einen anhaltenden Effekt der dama- ligen Hyposensibilisierung. Noch beeindruckender aber sind die Ergebnisse, die den Verlauf der Erkrankung betreffen: So waren in der Zwischenzeit sämtliche Kontrollpatienten gegenüber neuen Allergenen sensibilisiert, dagegen nur acht von 13 der SIT-Patienten. Von den ursprünglich neun Patienten in der aktiven Behandlungsgruppe mit polleninduziertem Asthma hatten nur noch drei Personen pollenassoziierte Beschwerden im unteren Respirationstrakt - gegenüber sieben von acht im konventionell behandelten Kollektiv.
Diese guten Langzeitresultate gehen nach Ansicht der Autoren auch auf die sorgfältige Patientenselektion zurück (rein saisonale Allergien, keine perenniale Erkrankung in der Anamnese).

\section{Fazit}

Die ermutigenden Resultate dieser wenn auch recht kleinen - Untersuchung passen zu experimentellen Daten, wonach der besondere Wirkansatz prophylaktische und lang anhaltende Effekte verspricht. Nach einer französischen Studie der Gruppe um Bousquet liegt hiermit eine weitere Arbeit vor, die belegt, dass Immuntherapie das Potenzial hat, den Verlauf einer allergischen Erkrankung zu modifizieren. Eine Immuntherapie über drei Jahre scheint ausreichend für eine lang anhaltende Wirksamkeit.

$w p a$

Eng PA et al. Long-term efficacy of preseasonal grass pollen immunotherapy in children. Allergy 2002; 57: 306-12 